\title{
The effect of quality of segmental bowel preparation on adenoma detection rate
}

Rui Guo 1,2,3,4, Yong-Jun Wang 1,2,3, Mo Liu', Jun Ge ${ }^{1,2,3}$, Ling-Ye Zhang ${ }^{1,2,3}$, Ling Ma ${ }^{1,2,3}$, Wen-Yu Huang ${ }^{1,2,3}$ and Hui-Hong Zhai ${ }^{1,2,3^{*}}$ (D)

\begin{abstract}
Background: The effectiveness in surveillance colonoscopy largely depends on the quality of bowel preparation. We aimed to investigate the quality of bowel preparation segmentally and its effect on Adenoma Detection Rate (ADR) and Advanced Adenoma Detection Rate (AADR) at corresponding bowel segments.

Methods: This is a single-centered and cross-sectional study. A consecutive of 5798 patients who underwent colonoscopy examination were included. Bowel preparation was evaluated based on Bowel Bubble Scale (BBS) in general and Boston Bowel Preparation Scale (BBPS) in each segment (right side, transverse and left side of colon) and total BBPS scores. The quality of bowel preparation was correlated with ADR and AADR.

Results: Four thousand nine hundred forty colonoscopies (14,820 bowel segments) were included in the final analysis. In which $30.9 \%$ scored $3,57.5 \%$ scored $2,11.2 \%$ scored 1 and $0.4 \%$ scored 0 on basis of BBPS. For each score, ADR were 10.8, 7.7, 4.9 and 3.2\%, respectively; whereas AADR were 4.5, 2.8,1.8 and 1.6\% $(P<0.05)$. 36.9\% of the colonoscopies showed presence of minimal bubbles and $34.3 \%$ with no bubble. For bowels without bubbles and with a large amount of bubbles, ADR were 28.3 and 20.0\% respectively; and AADR were 13.3 and $7.1 \%$ respectively.

Conclusions: Segmental bowels' cleanliness and the amount of bubbles in bowels significantly affect ADR and AADR. The better the bowel preparation at each segment is and the less bubbles in the bowel there are, the higher ADR and AADR we got. We suggest repeating colonoscopy if any segment of the bowel preparation is poor, or if there is more bubbles, even if the total score of BBPS indicates good or fair bowel preparation.
\end{abstract}

Keywords: Bowel preparation, Bowel bubble scale (BBS), Boston bowel preparation scale (BBPS), Adenoma detection rate (ADR), Advanced adenoma detection rate (AADR)

\section{Background}

Colorectal cancer is the 3rd most common cancer and the 4th leading cause of cancer-related mortality globally [1]. According to the National Cancer Report of China in 2015 , it is the 5th most common cancer in morbidity and mortality in China [2], and its incidence is rising each year. Surveillance colonoscopy is an important method of colorectal cancer screening. The effectiveness of adenoma detection in surveillance colonoscopy largely depends on the quality of colonoscopy [3-7]. There are many factors

\footnotetext{
* Correspondence: zhaihuihong@263.net

'Department of Gastroenterology, Beijing Friendship Hospital, Capital

Medical University, 95 Yongan Street, Xicheng Area, Beijing 100050, People's Republic of China

${ }^{2}$ Beijing Key Laboratory for Precancerous Lesion of Digestive Diseases, Beijing 100050, China

Full list of author information is available at the end of the article
}

contributing to good quality of colonoscopy, one of which is the quality of bowel preparation. Many studies proved the correlation of good bowel preparation to high adenoma detection rate (ADR) $[8,9]$. American College of Gastroenterology (ACG), American Gastroenterological Association (AGA), and American Society Gastrointestinal Endoscopy (ASGE) had jointly published a guideline on bowel preparation before colonoscopy to assist endoscopists in assessing the quality of bowel preparation objectively, and provide recommendations of various methods to optimize bowel preparation based on available clinical evidences [10-12].

Previous studies have focused almost exclusively overall cleanliness of the whole colon and rectum. Contrary to that, there are few studies focusing on the association of individual segment of bowel preparation with ADR

(c) The Author(s). 2019 Open Access This article is distributed under the terms of the Creative Commons Attribution 4.0 International License (http://creativecommons.org/licenses/by/4.0/), which permits unrestricted use, distribution, and 
and Advanced Adenoma Detection Rate (AADR). Our aim is to establish the evidence in correlating the quality of bowel preparation of the individual bowel segment to the ADR and AADR of the same bowel segment.

The presence of luminal bubble in colonoscopy may affect the ADR and AADR [13]. The routine use of simethicone during endoscopy procedure is not well established in China due to the lack of awareness of such practice. Assessing the effect of luminal bubble to ADR and AADR in the study may assist us in providing the evidence to recommend and justify the routine use of simethicone during the procedures in the country $[14,15]$.

\section{Methods}

\section{Study population}

5798 patients who underwent colonoscopy were included in the study. The exclusion criteria are: 1.Patients did not receive oral bowel cleaning agent (including cleansing enema or fasting). 2.Patients whose age was under 18 years old, patients with active psychiatric illness, patients who were incompetent in giving consent, multiple comorbidities with American Society of Anaesthesiology (ASA) class 3 or more, patients on anticoagulation which preclude biopsyprocedures, incomplete demographic data, withdrawal colonoscopy time $<6 \mathrm{~min}$, previous colectomy. 3.Failed completion of colonoscopy including poor bowel preparation (not able to identify the direction of the intestine), technical difficulty (physiologic morphological abnormality of colon, effect of intestinal peristalsis, patients with obesity), patients intolerant to colonoscopy procedure, pathological stricture or external compression leading to failure in completion of colonoscopy. This study was approved by Ethic Committee of Beijing Friendship Hospital. (Approval No. 2016-P2-107-01).

\section{Study design}

This is a single-centered and cross-sectional study. For each colonoscopy, the examination was done up to the caecum. Detailed examination of the colonic lumen was done during withdrawal, with assistance of flushing and suctioning aiming to cleanse the bowel. All examinations were performed using the CF-H260AI or CF-H260AL colonoscope, OLYMPUS CV26 processor and CLV260 light source.

All participating endoscopists fulfilled the requirements and credentials of standard guideline $[16,17]$. The endoscopists were permitted to use Narrow Band Imaging (NBI) or chromoendoscopy examination at their discretion to perform detailed characterization of polyps. Polyps were then examined by an appointed pathologist.

All participating endoscopists were trained including 8 min of Boston Bowel Preparation Scale (BBPS) educational video (http://domweb.bumc.bu.edu/bowelprep/), and have completed online assessment at the same website. These measures were undertaken to optimize the reliability, reproducibility and accuracy of the colonoscopic evaluation in the center.

Bowel preparations for all patients were done using standard split dose regimen of PEG (Shenzhen Wanhe Pharmaceutical Co. Ltd., Shenzhen, China). Patients were instructed to take low residue diet 1 day before colonoscopy. Then they received a total of $3 \mathrm{~L}$ of PEG. They were instructed to drink half of the volume the night before colonoscopy, and the other half $4-6 \mathrm{~h}$ before the procedure by a dose of $250 \mathrm{mls}$ every $10 \mathrm{~min}$ [18].

\section{Data collection}

Demographic and procedural data for each patient were collected including sex, age, time and date of colonoscopy, indication of colonoscopy, withdrawal time of colonoscopy, seniority of operator classified as senior, intermediate, and junior based on numbers of colonoscopy performed in the past ("senior" with lifetime colonoscopy experience of $>10,000$, "intermediate" of 5000 10,000 , "junior" of 1000-5000). Withdrawal time was defined as the time taken for withdrawal from caecum to anus while examining the colonic mucosa, inclusive of time used for washing and aspirating the bowel residues for colonic examination; the time taken for biopsy and polypectomy were not included in the withdrawal time.

The cleanliness scoring of each bowel segment examined (right side, transverse and left side of colon) was graded by BBPS. The BBPS involves assigning each of 3 regions of the above mentioned a score from 0 to 3 ( 0 as non-prepared and 3 as perfectly clean). Each segment score is summed for a total BBPS score ranging from 0 to 9 (score 9 as a perfectly clean colon and 0 as a nonprepared colon) [19]. At the same time, the amount of luminal bubbles was assessed based on Bowel Bubble Scale (BBS) (a scoring derived from capsule endoscopy examination) [20]. The amount of luminal bubbles was measured using a 4 points scale in which $0,1,2,3$ represent no air bubble, minimal bubbles ( $\leq 25 \%$ obscured view), moderate amount of air bubbles (25-50\% obscured view) and large amount of air bubbles ( $\geq 50 \%$ obscured view) respectively.

A detailed documentation for detected polyp(s) was done. The number, site, size (visual comparison with forceps of a known dimension and/or visualization after retrieval), morphology (pedunculated, sub-pedunculated, sessile or flat), method of polypectomy (forceps, snaring, endoscopic mucosal resection or endoscopic submucosal dissection), and pathological type of the polyp using narrow band imaging (NBI) or Chromoendoscopy technique, were all recorded.

Polyp(s) found during colonoscopy, along with the character of the lesion(s) were further classified into high risk and low risk as per international consensus: Low risk polyp was defined as $1-2$ adenomatous polyp with size $<10 \mathrm{~mm}$. High risk polyp was defined as $\geq 3$ adenomatous polyps, or 
any polyp size with $>10 \mathrm{~mm}$, or any polyp with more than $1 / 3$ volume of tubulovillous or high grade dysplasia [21].

\section{Statistical analysis}

Data were analyzed using SPSS19.0. Ages were described as mean \pm standard deviation (SD). ADR and AADR were described in percentage. Using a generalized estimating equations model, ADR and AADR of each group was adjusted based on sex, age, indication of colonoscopy, time of procedure, and seniority of endoscopists. Multiple comparisons were made between BBPS segment scores to detect the difference in ADR and AADR.95\% confidence interval and the $P$ value were evaluated. We use Chi-square to calculate the difference in ADR and AADR and ANOVA method to calculate the difference in BBPS and BBS, taking into consideration of seniority of endoscopists. $P$-value of $<0.05$ was considered as a statistically significant value.

\section{Results}

\section{Patient and colonoscopy characteristics}

There were 5798 patients who underwent colonoscopy during the study period. 875 patients were excluded based on the exclusion criteria. 4940 patients' colonoscopies (amount to 14,820 colon segments) were included in the final analysis (Fig. 1).
The mean age for the study population were $61.4 \pm$ 10.3 years-old. $48.2 \%(2380 / 4940)$ were male and $51.8 \%$ (2560/4940) were female. $84.7 \%(4186 / 4940)$ of the indications of colonoscopy were for screening, surveillance and diagnostic purposes and the remnants were done for therapeutic purposes including endoscopic resection of lesions and polypectomy. Lesions detected in 2115 colonoscopies (42.8\%). 1231 were adenomatous polyps (24.9\%) and 476 were advanced adenomatous polyps (9.6\%), (Table 1).

The colonoscopy examination was performed by 42 endoscopists. 31.9\% (1575/4940), 41.8\% (2063/4940) and $26.3 \%(1302 / 4940)$ of the colonoscopies were done by senior, intermediate, and junior endoscopists, respectively. ADR and AADR were up to $28.4 \%$ (301/1059) and 6.4\% $(68 / 1059)$ respectively in senior endoscopists group while they were $18.9 \%(245 / 1295)$ and $2.8 \%(36 / 1295)$ in junior group. The inter-observer difference in using BBPS and BBS scoring among different categories of endoscopists were negligible $(P=0.149)$. However, there were statistical difference in ADR $(P<0.001)$ and AADR $(P<0.001)$ between different categories of endoscopists based on seniority.

Primary Outcome: The effect of BBPS in segments of bowel towards ADR and AADR.

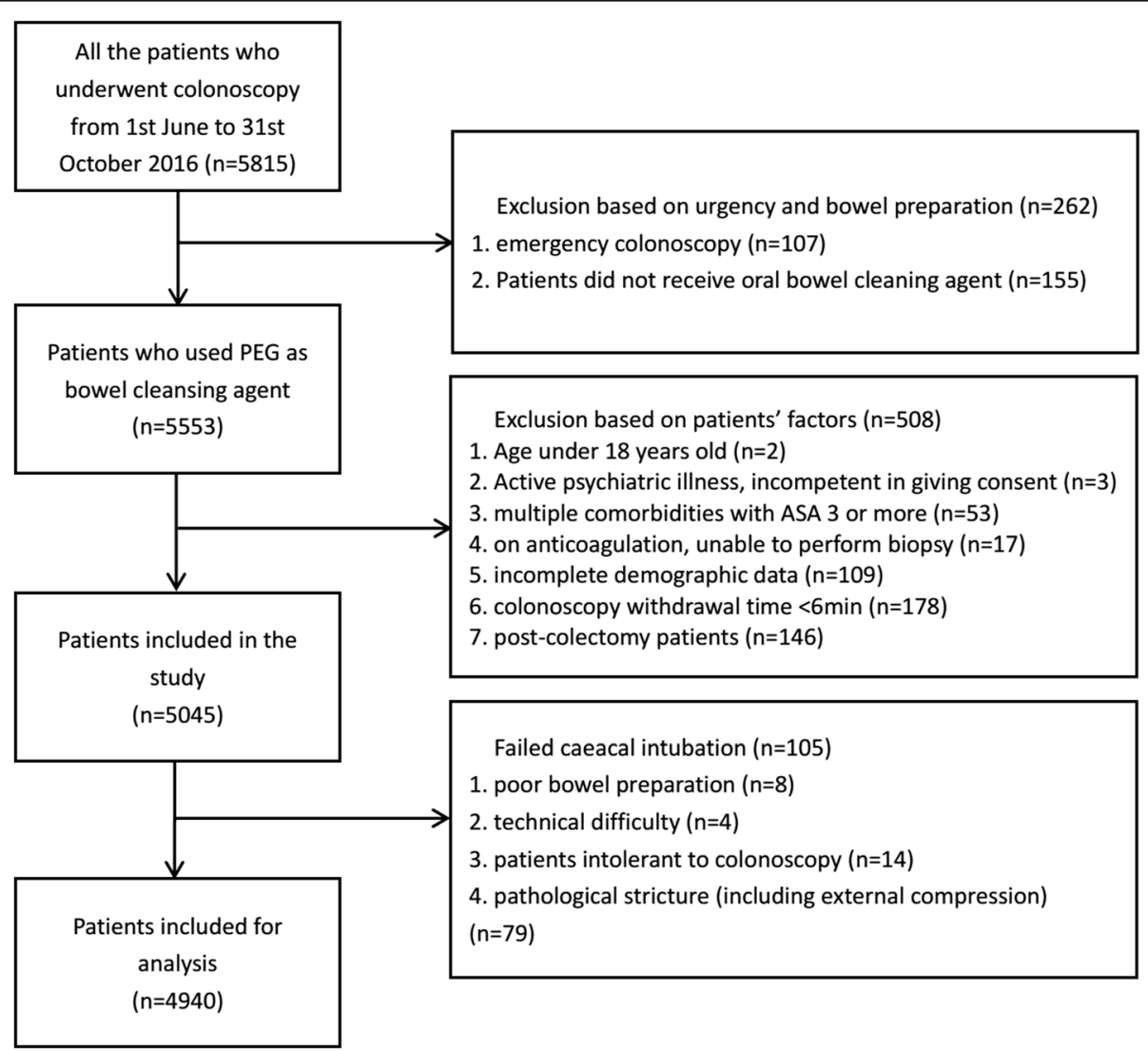

Fig. 1 Study Flow 
Table 1 Baseline Characteristics of the Study Participants

\begin{tabular}{ll}
\hline Patients,n & 4940 \\
\hline Age,years,mean \pm SD & $61.4 \pm 10.3$ \\
Male(\%) & $2380(48.2)$ \\
Indication(\%) & \\
Screening, Surveillance, Diagnostic & $4186(84.7)$ \\
For polypectomy & $314(6.4)$ \\
For ESD & $440(8.9)$ \\
Time of day colonoscopy performed(\%) & \\
Morning & $1236(25.0)$ \\
Afternoon & $3704(75.0)$ \\
Participating Endoscopists(\%) & \\
Senior & $1575(31.9)$ \\
Intermediate & $2063(41.8)$ \\
Junior & $1302(26.3)$ \\
Total BBPS score(\%) & \\
0-3(inadequate bowel preparation) & $101(2.1)$ \\
4-5(poor bowel preparation) & $885(17.9)$ \\
6(fair bowel preparation) & $1199(24.3)$ \\
7-8(good bowel preparation) & $111(2.2)$ \\
9(excellent or perfect bowel preparation) & $104(2.1)$ \\
Air bubble score(\%) & $2381(48.2)$ \\
0(no air bubble) & $374(7.6)$ \\
1(minimal bubbles) & \\
2(moderate amount of air bubbles) & $1231(24.9)$ \\
Collarge amount of air bubbles) & $1693(34.3)$ \\
Advancen & \\
Inflammator & \\
\hline
\end{tabular}

BBPS classified the bowel cleanliness based on the score of 0 to 3 from worst to excellent bowel preparation for colon segments. 30.9\% (4578/14820) scored 3, 57.5\% (8522/14820) scored 2, 11.2\% (1657/14820) scored 1 and $0.4 \%(63 / 14820)$ scored 0 for each score the ADR were $10.8 \%$ (493/4578), 7.7\% (655/8522), 4.9\% (81/1657) and $3.2 \%(2 / 63)$, respectively; whereas the AADR were $4.5 \%$ (207/4578), 2.8\% (239/8522), $1.8 \%$ (29/1657) and $1.6 \%$ $(1 / 63)(P<0.05)$, (Table 2).

In general, when comparing BBPS of individual segment higher score of BBPS shows significantly higher ADR and ADDR $(P<0.05)$. However, there were several control groups that did not show statistical significant difference in term of $\mathrm{ADR}$ and $\mathrm{AADR}$. At the right-sided colon, there was no difference in ADR between BBPS 2 and 3, AADR between BBPS 2 and 3, and AADR between BBPS 0 and 1 .

Secondary Outcome: The effect of overall BBPS and BBS towards ADR and AADR.

All the colonoscopy performed were given an overall BBPS from 0 to 9 (worst to excellent bowel preparation) for total colon by summing up the bowel segments' scores. The proportion from colonoscopy which scored from $\leq 3$ and $4-5,6,7,8$ and 9 were $2.1 \%$ (101/4940), 17.9\% (885/ 4940), 24.3\% (1199/4940), 32.8\% (1620/4940), 15.4\% (761/ $4940)$, and 7.6\% (374/4940) respectively. Most of the colonoscopies' BBPS scored 6 and above 80\% (3954/4940) and majority scored 7 . The higher the BBPS score was, the higher ADR and AADR we got.

The colonoscopies' BBPS score $\leq 3$ and ascending from 4 to 9 displayed rising ADR of $11.9 \%$ (12/101), $15.4 \%$ (38/246), 20.8\% (133/639), 21.8\% (261/1199), 27.8\% (450/1620), $28.2 \%(215 / 761)$, and $32.6 \%$ (122/374), respectively, and the difference was statistically significant (Chi-square value 59.217, $P<0.001$ ). Similarly, overall increasing AADR trend was observed from score of $\leq 3$ and ascending from 4 to 9 were $3.0 \%$ (3/101), 6.5\% (16/ 246), 5.5\% (35/639), 8.0\% (96/1199), 9.5\% (154/1620), $14.2 \%(108 / 761)$ and $17.1 \%(64 / 374)$, respectively, and the difference was statistically significant (Chi-square value 66.661, $P<0.001$ ) (Fig. 2).

Bowel bubble score were given for all the colonoscopies from 0 to 3 (no bubble in sight to $\geq 50 \%$ of view obscured by bubble). The proportion of BBS $0,1,2$, and 3 were $34.3 \%$ (1693/4940), 36.9\% (1824/4940), $19.9 \%$ $(984 / 4940)$ and $8.9 \%(439 / 4940)$. The lower the BBS score was (the less the luminal bubbles), the higher the ADR and AADR we got. The ADR of colonoscopy with BBS of $0,1,2$ and 3 were $28.2 \%$ (478/1693), 23.7\% (433/ 1824), $23.6 \%$ (232/984) and $20.0 \%(88 / 439)$ respectively. Similarly, the AADR of colonoscopy with BBS 0, 1, 2, and 3 were $13.3 \%$ (226/1693), 8.8\% (160/1824), 6.0\% (59/ 984 ) and $7.1 \%$ (31/439) respectively (Fig. 3).

\section{Discussion}

Optimizing bowel cleansing for colonoscopy is an important facet of successful colonoscopy surveillance programme. Guidelines recommend endoscopists to repeat colonoscopy when overall bowel preparation is inadequate because of high risk of missing polpys. The evidence to support this practice is scarce. Instead of focusing on overall bowel preparation, we assessed the importance of bowel cleanliness segmentally and correlate them with ADR and AADR of the same segments. In addition, we included overall colonoscopy bubble scoring in our study and correlate it with ADR and AADR in the similar manner. Our study found that the higher the segmental BBPS was (i.e. the better bowel preparation), the higher the ADR and AADR showed. Similarly, the 
Table 2 Detection Rates and Differences in Detection Rates for Different Levels of Preparation Quality Based on BBPS Segment Scores. Effect of Colonic Segments' BBPS on ADR

\begin{tabular}{|c|c|c|c|c|c|}
\hline \multicolumn{6}{|c|}{ A. Effect of Colonic Segments' BBPS on ADR } \\
\hline & Segments' score & ADR & Adjusted ADR & Comparison with BPPS & $\begin{array}{l}\text { Adjusted ADR difference } \\
(95 \% \mathrm{Cl})\end{array}$ \\
\hline \multirow[t]{5}{*}{ Right-sided colon } & $\mathrm{BBPS}=0$ & $2 / 56(3.6 \%)$ & $3.6 \%$ & BBPS2vs3 & $-0.4 \%(-0.9$ to $0.1 \%)$ \\
\hline & $\mathrm{BBPS}=1$ & $60 / 1214(4.9 \%)$ & $4.9 \%$ & BBPS1vs3 & $-2.3 \%(-2.8 \% \text { to- } 1.8 \%)^{*}$ \\
\hline & $\mathrm{BBPS}=2$ & $221 / 3243(6.8 \%)$ & $6.9 \%$ & BBPS1vs2 & $-1.9 \%(-2.1 \% \text { to-1.7\%) })^{*}$ \\
\hline & $\mathrm{BBPS}=3$ & $31 / 427(7.3 \%)$ & $7.3 \%$ & BBPSOvs2 & $-3.3 \%(-3.8 \% \text { to }-2.7 \%)^{*}$ \\
\hline & & & & BBPSOvs1 & $-1.4 \%(-2.2 \% \text { to- }-0.5 \%)^{*}$ \\
\hline \multirow[t]{5}{*}{ Transverse colon } & $\mathrm{BBPS}=0$ & $0 / 6(0.0)$ & $0.0 \%$ & BBPS2vs3 & $-0.9 \%(-1.3 \% \text { to- }-0.6 \%)^{*}$ \\
\hline & $\mathrm{BBPS}=1$ & $15 / 356(4.2 \%)$ & $4.2 \%$ & BBPS1vs3 & $-4.9 \%(-5.3 \% \text { to- }-4.5 \%)^{*}$ \\
\hline & $\mathrm{BBPS}=2$ & $279 / 3407(8.2 \%)$ & $8.2 \%$ & BBPS1vs2 & $-4.0 \%(-4.3 \% \text { to }-3.7 \%)^{*}$ \\
\hline & $\mathrm{BBPS}=3$ & 107/1171 (9.1\%) & $9.1 \%$ & BBPSOvs2 & $-8.2 \%(-8.4 \% \text { to- }-8.0 \%)^{*}$ \\
\hline & & & & BBPSOvs1 & $-4.2 \%(-4.5 \% \text { to- }-4.0 \%)^{*}$ \\
\hline \multirow[t]{4}{*}{ Left-sided colon (sigmoid-rectum) } & $\mathrm{BBPS}=0$ & $0 / 1(0.0 \%)$ & $0.0 \%$ & BBPS2vs3 & $-3.7 \%(-4.0 \%$ to-3.3\%) \\
\hline & $\mathrm{BBPS}=1$ & 6/87 (6.9\%) & $6.9 \%$ & BBPS1vs3 & $-5.0 \%(-5.7 \% \text { to- } 4.4 \%)^{*}$ \\
\hline & $\mathrm{BBPS}=2$ & 155/1872 (8.3\%) & $8.3 \%$ & BBPS1vs2 & $-1.4 \%(-2.1 \% \text { to- } 0.7 \%)^{*}$ \\
\hline & $\mathrm{BBPS}=3$ & $355 / 2980(11.9 \%)$ & $12.0 \%$ & & \\
\hline \multirow[t]{5}{*}{ All the colonic segments } & $\mathrm{BBPS}=0$ & $2 / 63(3.2 \%)$ & $3.2 \%$ & BBPS2vs3 & $-3.0 \%(-3.3 \% \text { to- } 2.9 \%)^{*}$ \\
\hline & $\mathrm{BBPS}=1$ & $81 / 1657(4.9 \%)$ & $4.9 \%$ & BBPS1vs3 & $-5.9 \%(-6.1 \% \text { to- } 5.7 \%)^{*}$ \\
\hline & $\mathrm{BBPS}=2$ & $655 / 8522(7.7 \%)$ & $7.7 \%$ & BBPS1vs2 & $-2.8 \%(-3.0 \% \text { to- } 2.6 \%)^{*}$ \\
\hline & $\mathrm{BBPS}=3$ & $49 / 4578(10.8 \%)$ & $10.8 \%$ & BBPSOvs2 & $-4.5 \%(-5.0 \% \text { to- }-4.0 \%)^{*}$ \\
\hline & & & & BBPS0vs1 & $-1.7 \%(-2.2 \% \text { to- } 1.2 \%)^{*}$ \\
\hline \multicolumn{6}{|c|}{ B. Effect of Colonic Segments' BBPS on AADR } \\
\hline & Segments' score & AADR & Adjusted AADR & Comparison with BPPS & $\begin{array}{l}\text { Adjusted AADR difference } \\
(95 \% \mathrm{Cl})\end{array}$ \\
\hline \multirow[t]{5}{*}{ Right-sided colon } & $\mathrm{BBPS}=0$ & $1 / 56(1.8 \%)$ & $1.8 \%$ & BBPS2vs3 & $-0.2 \%(-0.7$ to $0.3 \%)$ \\
\hline & $\mathrm{BBPS}=1$ & $21 / 1214(1.7 \%)$ & $1.7 \%$ & BBPS1vs3 & $-1.8 \%(-2.3 \% \text { to- } 1.3 \%)^{*}$ \\
\hline & $\mathrm{BBPS}=2$ & 107/3243 (3.3\%) & $3.3 \%$ & BBPS1vs2 & $-1.6 \%(-1.8 \% \text { to- } 1.3 \%)^{*}$ \\
\hline & $\mathrm{BBPS}=3$ & 15/427 (3.5\%) & $3.5 \%$ & BBPSOvs2 & $-1.5 \%(-2.0 \% \text { to- } 1.0 \%)^{*}$ \\
\hline & & & & BBPSOvs1 & $0.1 \%$ (- 0.6 to $0.7 \%)$ \\
\hline \multirow[t]{5}{*}{ Transverse colon } & $\mathrm{BBPS}=0$ & $0 / 6(0.0 \%)$ & $0.0 \%$ & BBPS2vs3 & $-0.9 \%(-1.1 \% \text { to- }-0.7 \%)^{*}$ \\
\hline & $\mathrm{BBPS}=1$ & $6 / 356(1.7 \%)$ & $1.7 \%$ & BBPS1vs3 & $-1.5 \%(-1.8 \% \text { to- } 1.2 \%)^{*}$ \\
\hline & $\mathrm{BBPS}=2$ & $77 / 3407$ (2.3\%) & $2.3 \%$ & BBPS1vs2 & $-0.6 \%(-0.8 \% \text { to- }-0.3 \%)^{*}$ \\
\hline & $\mathrm{BBPS}=3$ & $37 / 1171$ (3.2\%) & $3.2 \%$ & BBPSOvs2 & $-2.3 \%(-2.4 \% \text { to- } 2.2 \%)^{*}$ \\
\hline & & & & BBPSOvs1 & $-1.7 \%(-3.3 \% \text { to- }-0.0 \%)^{*}$ \\
\hline \multirow[t]{4}{*}{ Left-sided colon (sigmoid-rectum) } & $\mathrm{BBPS}=0$ & $0 / 1(0.0 \%)$ & $0.0 \%$ & BBPS2vs3 & $-2.3 \%(-2.6 \% \text { to- } 1.9 \%)^{*}$ \\
\hline & $\mathrm{BBPS}=1$ & $2 / 87(2.3 \%)$ & $2.3 \%$ & BBPS1vs3 & $-2.9 \%(-3.7 \% \text { to- } 2.1 \%)^{*}$ \\
\hline & $\mathrm{BBPS}=2$ & $55 / 1872(2.9 \%)$ & $2.9 \%$ & BBPS1vs2 & $-0.6 \%(-0.9 \% \text { to- }-0.3 \%)^{*}$ \\
\hline & $\mathrm{BBPS}=3$ & $155 / 2980(5.2 \%)$ & $5.2 \%$ & & \\
\hline \multirow[t]{5}{*}{ All the colonic segments } & $\mathrm{BBPS}=0$ & $1 / 63(1.6 \%)$ & $1.6 \%$ & BBPS2vs3 & $-1.7 \%(-1.9 \%$ to-1.5\%) \\
\hline & $\mathrm{BBPS}=1$ & 29/1657 (1.8\%) & $1.8 \%$ & BBPS1vs3 & $-2.8 \%(-3.0 \% \text { to- } 2.6 \%)^{*}$ \\
\hline & $\mathrm{BBPS}=2$ & 239/8522 (2.8\%) & $2.8 \%$ & BBPS1vs2 & $-1.1 \%(-1.2 \% \text { to- } 0.9 \%)^{*}$ \\
\hline & $\mathrm{BBPS}=3$ & $207 / 4578(4.5 \%)$ & $4.5 \%$ & BBPSOvs2 & $-1.2 \%(-1.6 \% \text { to- } 0.8 \%)^{*}$ \\
\hline & & & & BBPS0vs1 & $-0.2 \%(-0.7$ to $0.4 \%)$ \\
\hline
\end{tabular}




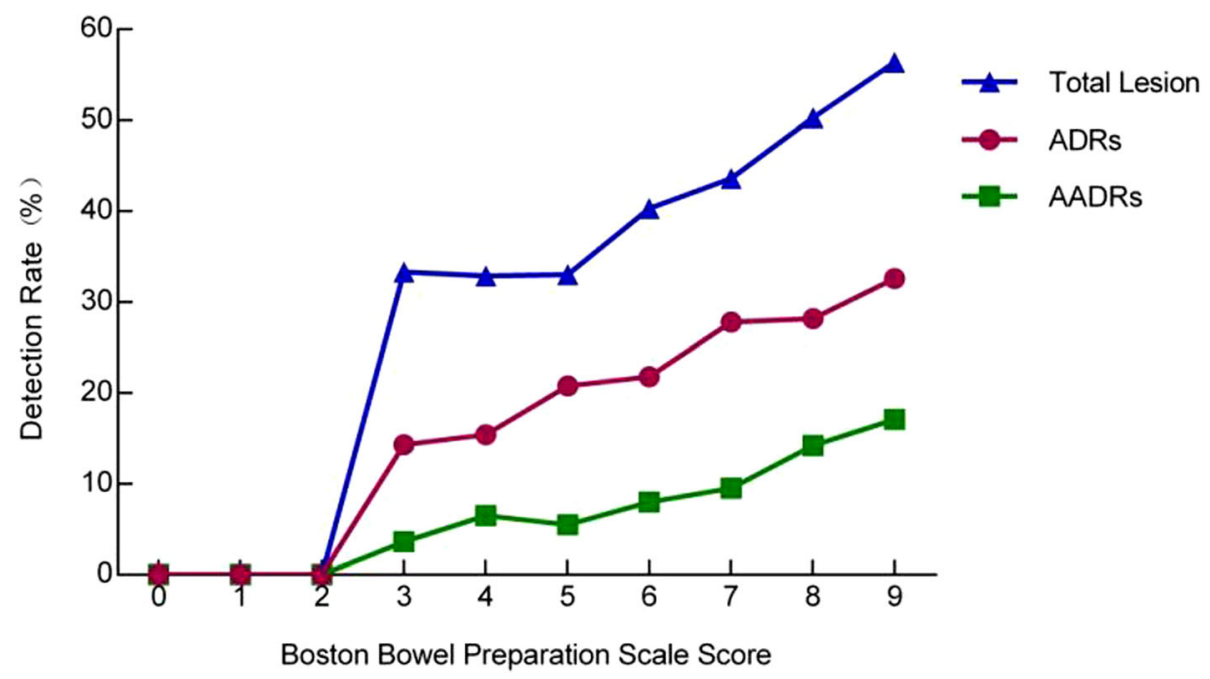

Fig. 2 Detection Rates For Total Lesion, Adenoma and Advanced Adenoma for Different Levels Based on BBPS. Note: The influence of BBPS total score (0-9) on detection rate of adenoma, high-risk adenoma and total lesions was statistically significant. $(P<0.001)$

lower the BBS score was (i.e. less bowel bubble), the higher the ADR and AADR were. Therefore we concluded that it would be beneficial to repeat colonoscopy to avoid missing adenomatous lesions when any segment of the BBPS is poor or contains large amount of bubbles, even overall bowel preparation is good or fair.

We used BBPS as standard assessment tool for bowel preparation. Comparing to Aronchick Bowel Preparation Scale and Ottawa Bowel Preparation Quality Scale, BBPS focuses on segmental assessment of bowel preparation. Moreover, it emphasizes on the assessment of bowel preparation after the attempt by endoscopists to cleanse bowel under endoscopy. Our study showed that when there is good bowel preparation with higher overall
BBPS score, the ADR and AADR are higher. Jain D et al [22] indicated that AADR for bowel preparation with BBPS 7-9 was $16.7 \%$, for BBPS $=4-6$ was $14.8 \%$ and for BBPS $=0-3$ was only $3.8 \%$. There was statistically significant difference for the 3 groups $(P<0.05)$. In 2009, Lai $E J$ et al [19] demonstrated the correlation of overall BBPS and polyp detection rate. The study found that when the total score of BBPS was $\geq 5$, the ADR was $40 \%$ and if the BBPS was $<5$, the ADR was only $24 \%(P<$ 0.02). Subsequently in 2010, Calderwood AH et al [23] introduced the BBPS for bowel segments' preparation assessment. This study verified the use of BBPS as an objective and reproducible tool in this field. At the same time, they concluded that higher BBPS segment scores

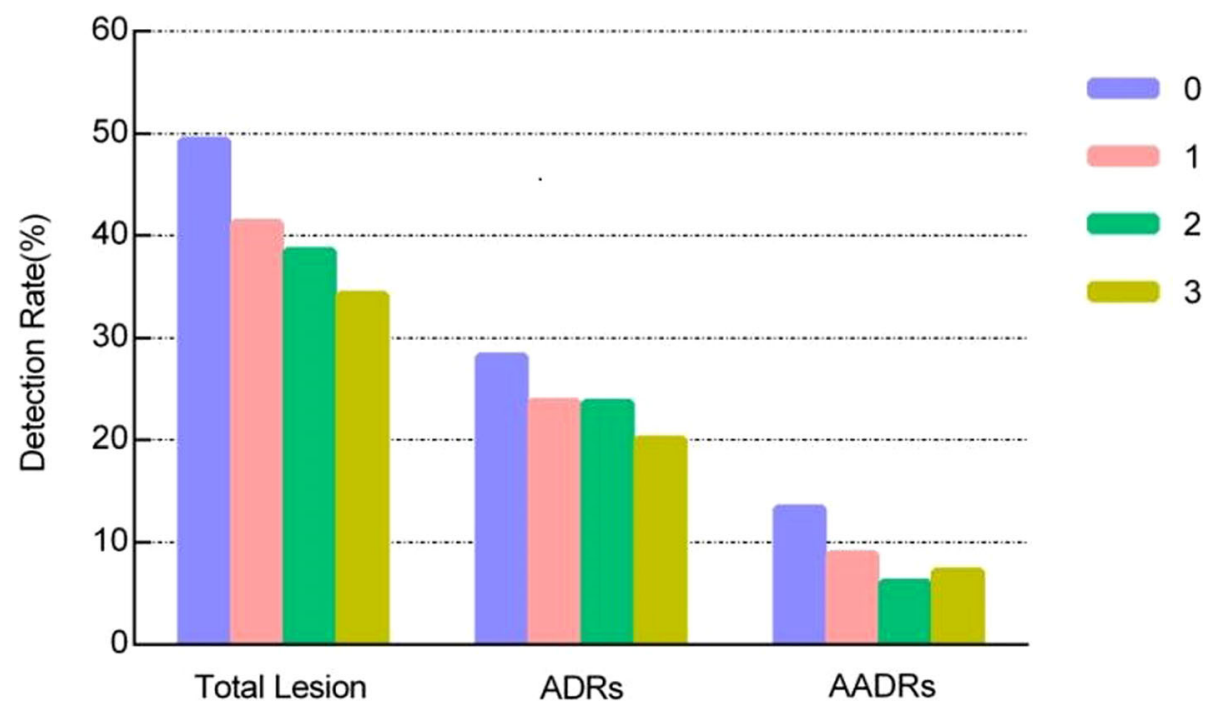

Fig. 3 Detection Rates for Total Lesion, Adenoma and Advanced Adenoma for Different Levels Based on BBS 
( 2 and 3 versus 0 and 1) were associated with improved polyp detection rate at the left colon (OR $2.58,95 \% \mathrm{CI}$ 1.34-4.98) and right colon (OR 1.60, 95\%CI 1.01-2.55). These results supported our primary outcomes.

To clarify the recommendation on follow-up for surveillance colonoscopy based on overall and segmental assessment of bowel preparation with BBPS score, Calderwood $\mathrm{AH}$ et al [24] conducted another study which showed that if the overall BPPS $\geq 6$ and individual bowel segment BBPS $\geq 2$, the bowel preparation were considered adequate and the follow-up colonoscopy should be planned according to the standard colonoscopy surveillance guideline. Meanwhile, if total scores $\leq 2$, it was regarded as inadequate and a repeated colonoscopy was highly recommended within 1 year. For colonoscopy with overall BBPS 3-5 (fair bowel preparation), Clark BT et al [25] had used the segmental bowel assessment to further decide the follow-up time for colonoscopy. The study particularly focused on the association of poor segmental bowel preparation with missed diagnosis of polyps. They concluded that patients with any colon segment score of $<2$ should take an early repeat colonoscopy whereas those with all segment score $\geq 2$ can repeat the surveillance colonoscopy based on standard guideline. In our study, when segmental bowel preparation (low BBPS score) were poor, ADR and AADR in the corresponding segment were low, and vice versa. Hence, we suggest repeating colonoscopy to reduce the risk of any polyp missing when the segments of bowel are poorly prepared.

Most of studies on bowel bubbles assessment were done on patients who underwent capsule endoscopy, with a standard bowel bubble score [20]. BBS has good reliability and reproducibility. Based on a previous study, $32-57 \%$ of colon had significant bowel bubbles which potentially obscure the view of endoscopy in China [18]. Compared to faecal materials and residues, bubbles are difficult to be cleared with conventional endoscopic washing and aspirating. We attempted to extrapolate the use of such score in large bowel examination and managed to demonstrate that it does affect ADR and AADR. We utilized BBS in addition to BBPS for bowel cleanliness assessment. In our study, 65.7\% (3247/4940) of patients had more or less bubbles that affect the quality of endoscopic examination. We found that the higher the BBS score was (more bubbles), the lower the ADR and AADR were. So it is imperative to repeat colonoscopy if the BBS score is high.

There were several limitations in our study. Firstly, the study did not restrict the selection of patients to those whom were colonoscopy naïve. These included the patient who had underwent previous colonoscopy and planned for therapeutic polypectomy or endoscopic resection. This would affect the ADR and AADR for the repeat cases obviously, as the lesion is known before the examination. Nevertheless, the statistical calculation in the study had focused on the association between bowel preparation and adenomatous lesion detection rather than discovery of these lesions per se. Furthermore, there is no evidence to show that bowel cleanliness is affected by the number of colonoscopy that was performed in the same patient. We believe that the inclusion of the repeat cases in the study had minimal effect on the correlation.

Secondly, the inadequacy in number of study population in certain categories has resulted in failure in demonstrating the difference on the ADR and AADR in a few groups. If the number of patients had been greater, it might have been able to show a difference, which large sample studies are needed for further testified.

Thirdly, the assessment of BBS score can be improved by performing segmentally and by using simethicone $[14,15]$. We have observed that colonic bubbles mainly occurred in right colon and transverse colon and it is well-established that polyp missing rate, especially sessile lesion which carries high risk of malignant transformation, is unacceptably high in this segment of colon. Meanwhile, it is ideal to use simethicone as suggested by several guidelines to reduce luminal bubbles in order to improve the endoscopic view. However, simethicone is not a routine in China, particularly the awareness of its importance is not widespread. As a result, the data that we obtained in the study reflected the real-world practice locally. Thus we believe that a follow up study assessing the bubble scoring segmentally, with further intervention to reduce luminal bubbles should be done to demonstrate the significant correlation of reducing luminal bubbles with improvement of ADR/AADR.

Finally, this is a single-centered and cross-sectional study. Despite of the statistical adjustment, there was inherited bias due to the existence of many other confounding factors. This can be improved by conducting a multi-centered prospective trial.

\section{Conclusions}

Our study illustrates that the cleaner (the higher BBPS score) the individual segment of bowel was, and the less amount of overall luminal bowel bubbles existed (the lower the BBS score), the higher ADR and AADR were. We concluded that a repeat colonoscopy should be planned if any segment of the bowel is poorly prepared, or if there is more bubbles, even if the overall assessment score of the bowel preparation is good or fair.

\section{Abbreviations}

AADR: Advanced Adenoma Detection Rate; ACG: American College of Gastroenterology; ADR: Adenoma Detection Rate; AGA: American

Gastroenterological Association; ASA: American Society of Anaesthesiology; ASGE: American Society Gastrointestinal Endoscopy; BBPS: Boston Bowel Preparation Scale; BBS: Bowel Bubble Scale; EMR: Endoscopic Mucosal Resection; ESD: Endoscopic Submucosal Dissection; NBI: Narrow Band Imaging; PEG: Polyethylene Glycol-electrolyte Solution 


\section{Acknowledgements}

We would like to thank Dr. Gew Lai Teck for providing the modifications to the language of the article.

\section{Authors' contributions}

RG: analyzed and interpreted the patient data, and was a major contributor in designing the study and writing the manuscript. YJW: analyzed and interpreted the patient data. ML: made contributions to data acquisition and data statistics. JG: collected and analyzed the patient data regarding the effect of BBPS on ADR. LYZ: collected and analyzed the patient data regarding the effect of BBS on ADR. LM: analyzed and interpreted the patient data regarding the effect of BBPS on ADR. WYH: analyzed and interpreted the patient data regarding the effect of BBS on ADR. HHZ: designed the study and revised the manuscript. Statement: All authors read and approved the final manuscript.

\section{Funding}

Our study was supported by National Key Technologies R\&D ProgramNo.2015BAl13B09. We paid the staff of the endoscopy center to collect patients' data and endoscopic data (evaluate BBS and BBPS scoring and make diagnosis); two statisticians were hired for statistical analysis; we paid Dr. Gew Lai Teck to polish the article.

\section{Availability of data and materials}

All data generated or analysed during this study are included in this published article. The datasets generated and analysed during the current study are available from the corresponding author by email zhaihuihong@263.net on reasonable request.

\section{Ethics approval and consent to participate}

This study was approved by Ethic Committee of Beijing Friendship Hospital, and this Ethic Committee comply with the Declaration of Helsinki (Approval No. 2016-P2-107-01).

This is a retrospective study. The ethics committee agreed to exempt the informed consent. According to the China Health Committee issued "Ethical Review of Biomedical Research involving Human Beings" (2016), thirty-ninth: on the identity information of human material or data, has been unable to find the subjects, and the research project is not related to personal privacy and commercial interests, can be exempt from informed consent.

\section{Consent for publication}

Not applicable. Our manuscript does not contains any individual person's data in any form (including individual details, images or videos).

\section{Competing interests}

The authors declare that they have no competing interests.

\section{Author details}

'Department of Gastroenterology, Beijing Friendship Hospital, Capital Medical University, 95 Yongan Street, Xicheng Area, Beijing 100050, People's Republic of China. ${ }^{2}$ Beijing Key Laboratory for Precancerous Lesion of Digestive Diseases, Beijing 100050, China. ${ }^{3}$ National Clinical Research Center for Digestive Diseases, Beijing 100050, China. ${ }^{4}$ Department of Gastroenterology, Beijing Shijingshan Hospital, Teaching Hospital of Capital Medical University, Beijing 100043, China. ${ }^{5}$ National Clinical Research Center of Digestive Diseases, Beijing Friendship Hospital, Capital Medical University, Beijing 100050, China.

Received: 25 March 2018 Accepted: 10 June 2019

Published online: 08 July 2019

\section{References}

1. Torre LA, Bray F, Siegle RL, Ferlay J, Lortet-Tieulent J, Jemal A. Global Cancer statistics, 2012. CA Cancer J Clin. 2015;65(2):87-108.

2. Chen W, Zheng R, Baade PD, Zhang S, Zeng H, Bray F, et al. Cancer statistics in China, 2015. CA Cancer J Clin. 2016;66(2):115-32.

3. Zauber AG, Winawer SJ, O'Brien MJ, Lansdorp-Vogelaar I, van Ballegooijen M, Hankey BF, et al. Colonoscopic polypectomy and long-term prevention of colorectal-cancer deaths. New Engl J Med. 2012;366(8):687-96.

4. Corley DA, Jensen CD, Marks AR, Zhao WK, Lee JK, Doubeni CA, et al. Adenoma detection rate and risk of colorectal Cancer and death. New Engl J of Med. 2014;370(14):1298-306.
5. Doubeni CA, Corley DA, Quinn VP, Jensen CD, Zauber AG, Goodman M, et al. Effectiveness of screening colonoscopy in reducing the risk of death from right and left colon cancer: a large community-based study. Gut. 2018 Feb;67(2):291-8.

6. Kahi CJ, Rex DJ, Imperiale TF. Screening, surveillance, and primary prevention for colorectal cancer: a review of the recent literature. Gastroenterology. 2008;135(2):380-99.

7. Nishihara R, Wu K, Lochhead P, Morikawa T, Liao X, Qian ZR, et al. Longterm colorectal-cancer incidence and mortality after lower endoscopy. N Engl J Med. 2013;369(12):1095-105.

8. Lebwohl B, Kastrinos F, Glick M, Rosenbaum AJ, Wang T, Neugut Al. The impact of suboptimal bowel preparation on adenoma miss rates and the factors associated with early repeat colonoscopy. Gastrointest Endosc. 2011; 73(6):1207-14.

9. Clark BT, Rustaqi T, Laine L. What level of bowel prep quality requires early repeat colonoscopy: systematic review and meta-analysis of the impact of preparation quality on adenoma detection rate. Am J Gastroenterol. 2014; 109(11):1714-23.

10. Rex DK, Schoenfeld PS, Cohen J, Pike IM, Adler DG, Fennerty MB, et al. Quality indicators for colonoscopy. Gastrointest Endosc. 2015;81 (1):31-53.

11. Lieberman DA, Rex DK, Winawer SJ, Giardiello FM, Johnson DA, Levin TR. Guidelines for colonoscopy surveillance after screening and polypectomy: a consensus update by the US multi-society task force on colorectal Cancer. Gastroenterology. 2012;143(3):844-57.

12. Johnson DA, Barkun AN, Cohen LB, Dominitz JA, Kaltenbach T, Martel M, et al. Optimizing adequacy of bowel cleansing for colonoscopy: recommendations from the US multi-society task force on colorectal Cancer. Gastroenterology. 2014;147(4):903-24.

13. Yeh JH, Hsu MH, Tseng CM, Chen TH, Huang RY, Lee $C T$, et al. The benefit of adding oral simethicone in bowel preparation regimen for the detection of colon adenoma: a systematic review and meta-analysis. J Gastroenterol Hepatol. 2019;34(5):830-6.

14. Bai Y, Fang J, Zhao SB, Wang D, Li YQ, Shi RH, et al. Impact of preprocedure simethicone on adenoma detection rate during colonoscopy: a multicenter, endoscopist-blinded randomized controlled trial. Endoscopy. 2018;50(2): 128-36. https://doi.org/10.1055/s-0043-119213.

15. Zhang S, Zheng D, Wang J, Wu J, Lei $P$, Luo Q, et al. Simethicone improves bowel cleansing with low-volume polyethylene glycol: a multicenter randomized trial. Endoscopy. 2018;50(4):412-22.

16. Rembacken B, Hassan C, Riemann JF, Chilton A, Rutter M, Dumonceau JM, et al. Quality in screening colonoscopy: position statement of the European Society of Gastrointestinal Endoscopy (ESGE). Endoscopy. 2012;44(10):957-68.

17. Rex DK, Schoenfeld PS, Cohen J, Pike IM, Adler DG, Fennerty MB, et al. Quality indicators for colonoscopy. Am J Gastroenterol. 2015;110(1):72-90.

18. Chinese Society of Digestive Endoscopy. Guidelines for the preparation of digestive endoscopy in China (Draft). Chinese Journal of Digestion. 2013; 19(9):354-6.

19. Lai EJ, Calderwood AH, Doros G, Fix OK, Jacobson BC. The Boston bowel preparation scale: a valid and reliable instrument for colonoscopy-oriented research. Gastrointest Endosc. 2009;69(3 Pt 2):620-5.

20. McNally PR, Maydonovitch CL, Wong RK. The effectiveness of simethicone in improving visibility during colonoscopy: a double-blind randomized study. Gastrointest Endosc. 1988;34(3):255-8.

21. Chinese Society of Digestive Endoscopy. The diagnosis and treatment of early colorectal cancer and precancerous lesions in China(Chongqing,2014). Chinese Journal of Digestive Endoscopy. 2015;2:69-85.

22. Jain D, Momeni M, Krishnaiah M, Anand S, Singhal S. Importance of reporting segmental bowel preparation scores during colonoscopy in clinical practice. World J Gastroenterol. 2015;21(13):3994-9.

23. Calderwood AH, Jacobson BC. Comprehensive validation of the Boston bowel preparation scale. Gastrointest Endosc. 2010 Oct;72(4):686-92.

24. Calderwood AH, Schroy PC 3rd, Lieberman DA, Logan JR, Zurfluh M, Jacobson BC. Boston bowel preparation scale scores provide a standardized definition of adequate for describing bowel cleanliness. Gastrointest Endosc. 2014;80(2):269-76.

25. Clark BT, Protiva P, Naqar A, Imaeda A, Ciarleqlio MM, Deng Y, et al. Quantification of adequate bowel preparation for screening or surveillance colonoscopy in men. Gastroenterology. 2016;150(2):396-405.

\section{Publisher's Note}

Springer Nature remains neutral with regard to jurisdictional claims in published maps and institutional affiliations. 